\title{
Analysis and Design of a New Dual Band Microstrip Patch Antenna Based on Slot Matching Y-Shaped
}

\author{
R. Walia, S. Ghnimiª, A.G. Hand ${ }^{\mathrm{b}}$ and T. Razban ${ }^{\mathrm{b}}$ \\ ${ }^{a}$ High Frequency Electronics Circuits and Systems, Science Faculty of Tunis, University Tunis El Manar, Tunis, Tunisia \\ ${ }^{b}$ IETR Polytech Nantes, Universite de Nantes, site de la Chantrerie, rue Christian-Pauc, Nantes, France
}

Received 4 June 2013; accepted 30 December 2014

\begin{abstract}
A new compact microstrip slot antenna with Y-shaped coupling aperture is proposed. This antenna is based on a rectangular form with a microstrip fed line for excitation, and consists of slots on the edge of the radiation patch to provide dual-band operation. The design and simulation of the antenna were performed using CST Microwave Studio simulator. A prototype of the proposed antenna has been constructed and tested. In order to validate the return loss of the prototype antenna, the experimental results are presented. The results show two available bands with $-10 \mathrm{~dB}$ band $\mathrm{S}$ at 2.7 $\mathrm{GHz}$ and band $\mathrm{C}$ at $5 \mathrm{GHz}$. Also, good radiation performance and antenna gain over the two frequency ranges have been obtained. Computation results confirm the experimental findings.
\end{abstract}

Keywords: Microstrip antenna, Dual band antenna, Slot antenna, CST microwave studio simulator.

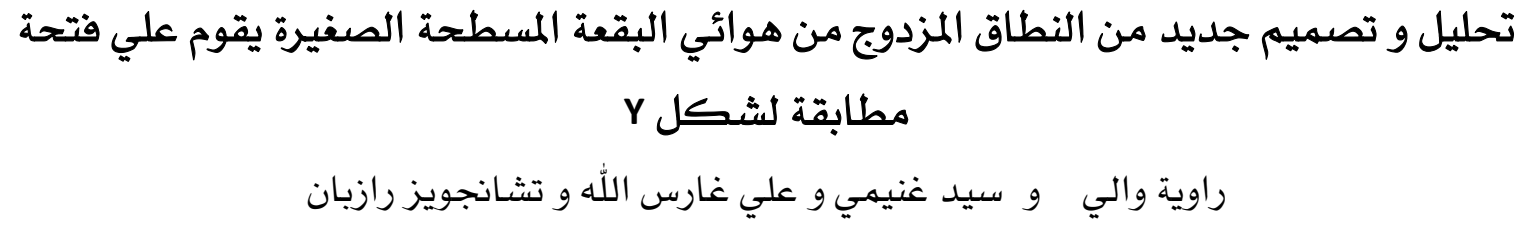

*Corresponding author's e-mail: said.ghnimi@fst.rnu.tn 


\section{Introduction}

The demands on radar application performance have increased rapidly over the last few years. Future requirements for advanced radar antennas include the need for dual-band operation (Allen et al. 2005; Christina et al. 2009; Zhilong et al. 2012; Qu et al. 2006; Andan et al. 2009; Khunead et al. 2007; Khaled and Saad 2008; Abdelaziz 2006; Gai et al. 2010; Saidatul et al. 2009; Anden et al. 2010; Balanis 1997 AND Ghnimi et al. 2013). It is essential to integrate multiple bands for use in one device. In the literature, numerous antennas for dual band radar applications have been used. In (Allen et al 2005), a dual tapered meander slot antenna is designed for use as an element in a phased array antenna system. In (Christina et al. 2009), a novel broadband monopole antenna designs with dual-band circular polarization are presented. In (Zhilong 2012), the author presents a method for reducing radar cross section of a patch antenna in two frequency bands. This method is based on the metamaterial composed of double-square-loop structure, which is loaded around the exciting source. In (Qu et al. 2006), a novel dual-band dual-polarized micro-strip antenna array is presented. In Adnan et al. 2009), a loop-loaded printed dipole antenna array is introduced for a dual-band radar application operating at 3.0/5.5 $\mathrm{GHz}$ bands. In (Khunead et al. 2007), a rectangular slot antenna composed of two conductor strips located in a slot cut in the ground plane was investigated for dual frequency.

Several approaches are developed in order to design multi-band micro-strip patch antennas such as slot matching concept (Khaled and Saad 2008), as two different radiating elements connected together through a matched section in Abdelaziz 2006), as a pair of U-shaped strips in (Gai et al. 2010), as a Fractal planar inverted F antenna in (Saidatul et al. 2009).

In this paper, the proposed antenna presents a simple technique; indeed the use of a slot on the patch with a simple substrate of low cost and larger height allows a good performance.
The main purpose of the antenna designing is to obtain a directional radiation and a high gain with widest bandwidth as much as possible. The organization of this paper is as follows: in section 2; the design of the rectangular microstrip patch antenna is given. The parametric study for this proposed antenna is presented in section 3; the fabrication and the measurement of S-parameter, radiation pattern and gain for validation are in section 4 . Section 5, contains conclusions and recommendations for further studies.

\section{Antenna Design}

Designing a simple patch antenna involves the choice of materials before calculating the dimensions of the patch and the width of the feed line (which depends on the input impedance). All are simulated using CST Microwave Studio software. The model is then adjusted and optimized for best results. Antennas for radar applications must have a large bandwidth and to ensure this condition, the substrate should be thick and having low dielectric constant. This ensures the desirable qualities; better efficiency, larger bandwidth and better radiation (Balanis 1997). This process provides a reasonably accurate starting point but it does not provide the final dimensions of the patch (Balanis 1997). The dimensions of the antenna for a chosen resonant frequency can be calculated using Cavity Model (Saidatul et al. 2009).

A prototype of the antennas was designed (Fig. 1) and its characteristics is verified with theory and simulation results. The structure of this antenna is configured to cover the band $S$ for radar application $(2 \mathrm{GHz}-4 \mathrm{GHz})$. The studied antenna is printed on the FR4_Epoxy which is a very commonly used substrate, which allows a slight compromise between size and losses of the antenna. The metallization level is $t$ $=0.035 \mathrm{~mm}$ with a dielectric loss $\tan \delta=0.002$, a relative permittivity $\varepsilon_{\mathrm{r}}=4.6$ and $\mathrm{a}$ thickness $\mathrm{h}=1.58 \mathrm{~mm}$. 


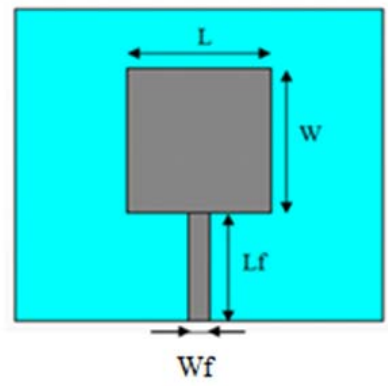

(a)

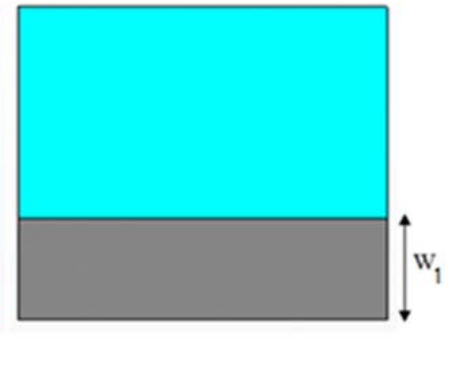

(b)

Figure 1. Geometry of the proposed antenna: (a) Top view, (b) Side view.

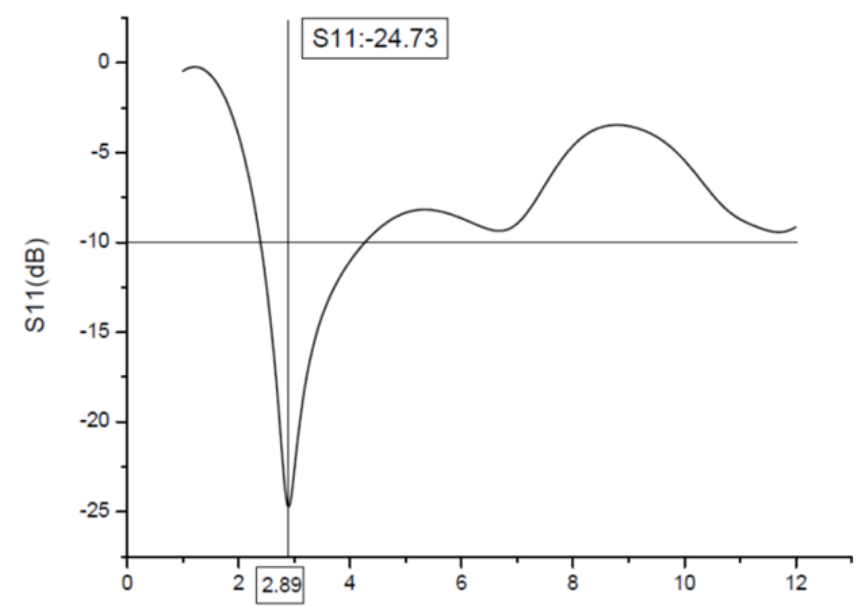

Figure 2. Simulated reflection coefficient as a function of frequency.

The transverse dimension of this antenna is $50 \times 45 \mathrm{~mm}^{2}$. The patch size is $21 \times 19.5 \mathrm{~mm}^{2}$, and it is excited by a feed line with dimension $15.5 \times 3 \mathrm{~mm}^{2}$. The calculations are done for a patch antenna with a ground plane. By removing a part of the ground plane and reducing it to a dimension $\mathrm{w}_{1}=14.5 \mathrm{~mm}$ printed on a portion of the underside of the substrate provide an increase of the bandwidth.

After the simulation of this antenna with dimensions given above, we found the reflection coefficient, as a function of the frequency (Fig. 2).

It can be seen from Fig. 2 that the antenna resonates at $2.89 \mathrm{GHz}$ with a minimum reflection coefficient of $-24.73 \mathrm{~dB}$. The reflection coefficient is less than $-10 \mathrm{~dB}$ between 2.4 and 4.26 GHz. This bandwidth obtained for the optimized structure covers the S-band radar applications.

\section{Parametric Study of Dual Band Antenna}

Our objective is essentially the design of a dual-band antenna to cover two radar bands. Indeed, several techniques are used including the disturbance slot geometry in the radiating element, which allows the creation of new resonances (Adnen et al. 2010 and Ghnimi et al. 2013). Based on this idea, we created slots in the simulated antenna geometries given in the previous section. The band that we want to add with the insertion of a slot in the radiating element is the $\mathrm{C}$ band radar applications which uses frequencies ranging from 4 to 8 $\mathrm{GHz}$, while maintaining the first band around $3 \mathrm{GHz}$ (Fig. 3). 


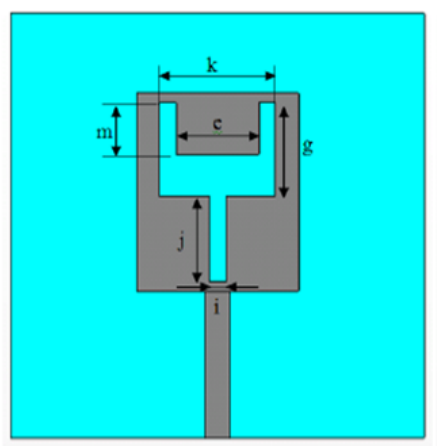

(a)

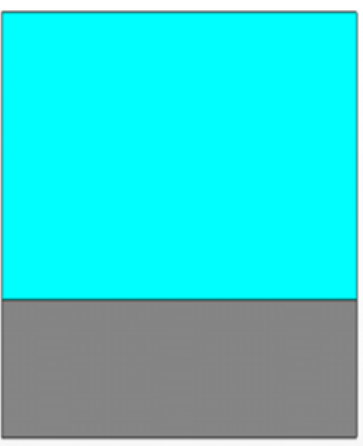

(b)

Figure 3. Geometry of the dual band antenna and slot parameters: (a) Top view, (b) Side view.

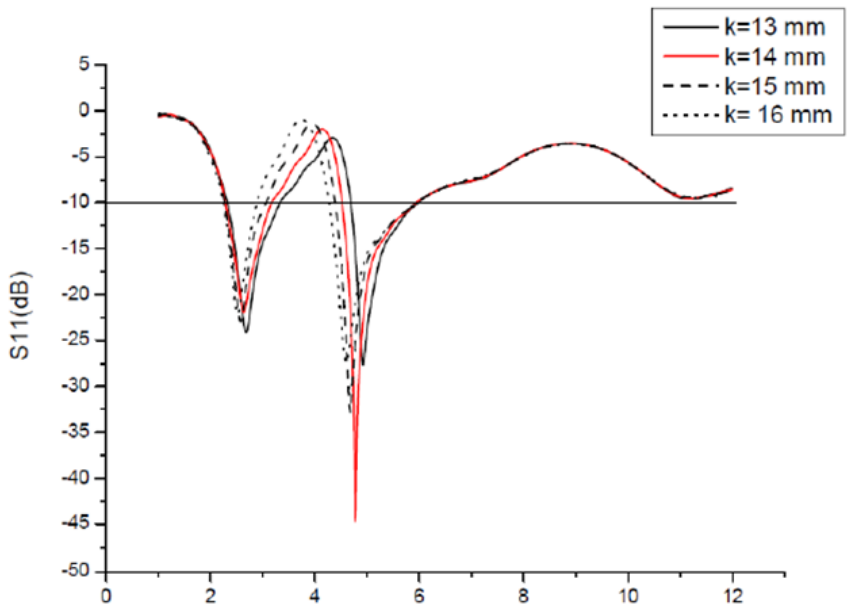

Figure 4. Simulated reflection coefficient of the antenna with slot for different values of ' $k$ '.

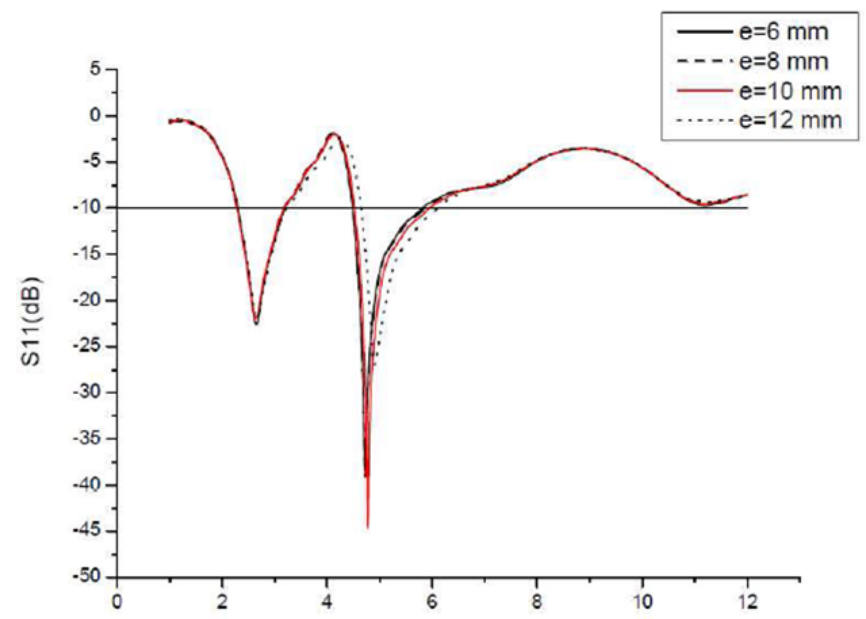

Figure 5. Simulated reflection coefficient of the antenna with slot for different values of ' $e$ '. 


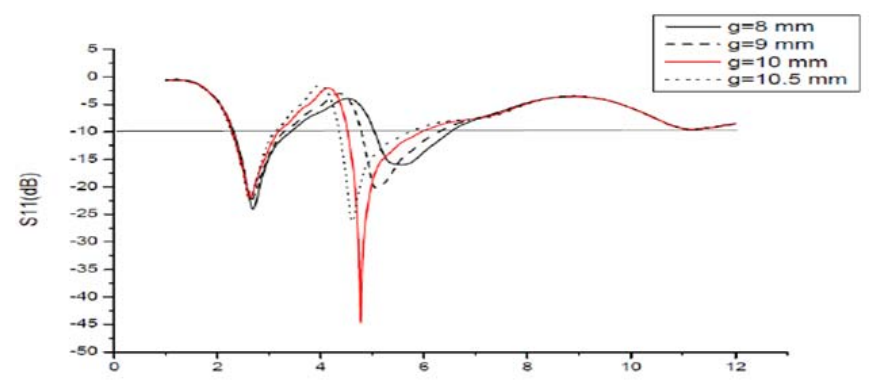

Frequency (GHz)

Figure 6. Simulated reflection coefficient of the antenna with slot for different values of ' $g$ ' .

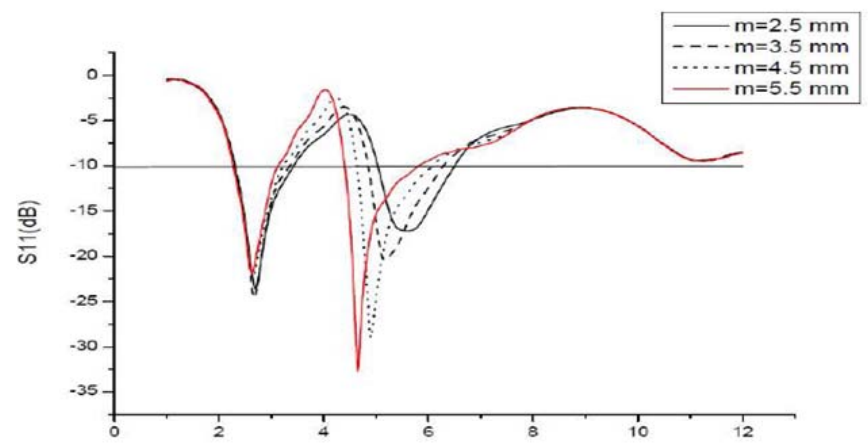

Frequency $(\mathrm{GHz})$

Figure 7. Simulated reflection coefficient of the antenna with slot for different values of ' $m$ '.

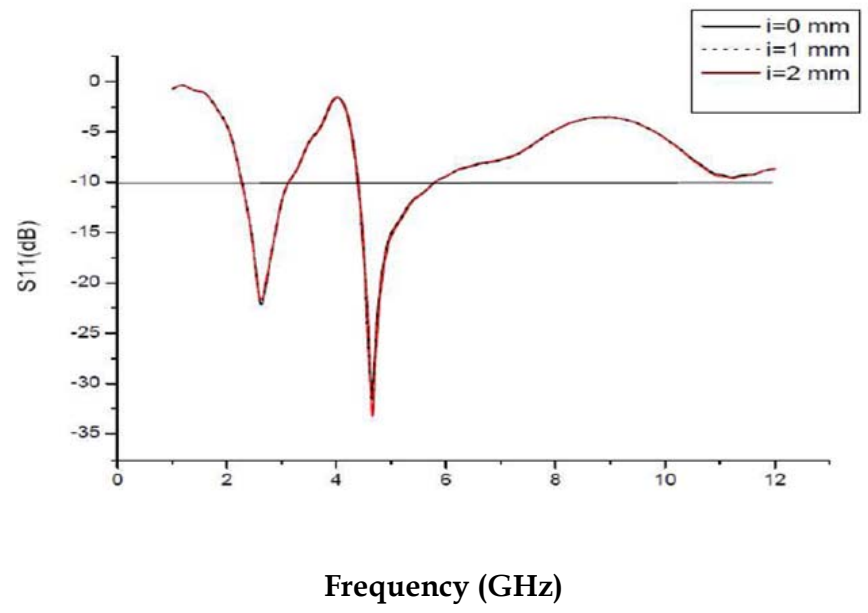

Figure 8. Simulated reflection coefficient of the antenna with slot for different values of ' $i$ '.

After insertion of the slot, we are interested in optimizing the printed antenna for a radar antenna in both bands. To do this, we used a parametric study of slots dimensions to see 
which one affects the most the performances of the proposed antenna. The dimensions of the proposed antenna are chosen to obtain the lowest return loss and the widest bandwidth and one parameter is changed at a time while the others are kept constant. Figures 4 to 8 show the parametric effect of the slot on electrical characteristics of the proposed antenna. All the results in these figures show that this antenna has two resonant frequencies (C and $\mathrm{S}$ ) for radar application.

It can be seen from Fig. 4 that with an increase in the parameter ' $k$ ', the bandwidth of the lower band decreases, while the upper resonant frequency shifts down. On the other side the parameter ' $\mathrm{e}$ ' does not affect the impedance characteristics significantly, as shown in Fig. 5. However, a slight degradation in performance at the upper frequencies can be observed. However return loss decreases by $4 \mathrm{~dB}$ when e increases from $6 \mathrm{~mm}$ to $10 \mathrm{~mm}$ at $\mathrm{f}=4.8 \mathrm{GHz}$. That is why, the choice of $\mathrm{k}=14$ $\mathrm{mm}$ and $\mathrm{e}=10 \mathrm{~mm}$ are the most appropriate for the best performance.

Figures 6 and 7 show that the variation of ' $\mathrm{g}$ ' and ' $\mathrm{m}$ ', allows us to have a very significant effect on the coverage of the second bandwidth of the antenna and its adaptation. Indeed, when the parameters ' $\mathrm{m}$ ' and ' $\mathrm{g}$ ' increase, the reflection coefficient in the second band increases and the resonant frequency decreases. Thus, for a better performance, it is advisable to choose $\mathrm{m}=5.5 \mathrm{~mm}$ and $\mathrm{g}=10 \mathrm{~mm}$.

The parameter ' $i$ ' as shown in Fig. 8, is opposite to the aforementioned effect of the other parameters. Indeed, we note that the bandwidth is not influenced by the different values of 'i'. However, the reflection coefficient is slightly affected in the second band.

From the point of view of the resonant frequencies, the microstrip patch antenna is considered a single resonant cavity functioning mainly at the band S. But, with a good choice of the $\mathrm{Y}$ shaped slot dimension; we can give other possibility to cover other $\mathrm{C}$ radar bands.

Following the simulation of the optimized antenna with the final dimensions of the slot, we were able to get a dual-band aspect for better performance of the antenna (Fig. 9). It covers both the first band from $2.27 \mathrm{GHz}$ to $3.13 \mathrm{GHz}$ lying in the $S$ band radar and the second band between $4.4 \mathrm{GHz}$ and $5.8 \mathrm{GHz}$ which is in the band $\mathrm{C}$ for radar application. Moreover, the antenna admits respectively a reflection coefficient of $-21.7 \mathrm{~dB}$ at $2.63 \mathrm{GHz}$ and $4.65 \mathrm{GHz}$ $-33.22 \mathrm{~dB}$ at the first and second operating bands.

\section{Simulated and Measured Results}

To verify the validity of the analysis presented in the previous section, measurements were performed to study the electric and radiation characteristics for the proposed antenna.

Figure 10 shows the prototype of the performed monopole dual band antenna. It is printed on a FR4_Epoxy substrate with dimensions of $50 \times 45 \mathrm{~mm}^{2}$, the relative permittivity $\varepsilon_{\mathrm{r}}=4.6$ and a thickness $\mathrm{h}=1.58$ $\mathrm{mm}$.

\subsection{Measured Reflection Coefficient}

Figure 11 illustrates the evolution of the reflection coefficient simulated and measured within the frequency band of $1-12 \mathrm{GHz}$.

According to Fig. 11, we observe a good agreement between the simulated and measured reflection coefficients around the $\mathrm{S}$ band. However there is a slight difference in $\mathrm{C}$ band. This confirms that the variation of the second band is affected by the insertion of the slot.

Indeed, the variation of the measured reflection coefficient shows two frequency bands: The low-band resonant frequency is located at about $2.7 \mathrm{GHz}$ with $-10 \mathrm{~dB}$ impedance bandwidth from about 2.3 to $2.5 \mathrm{GHz}$. However, the high-band resonant frequency is located at about $5 \mathrm{GHz}$ with $-10 \mathrm{~dB}$ impedance bandwidth from about 4.9 to $6.0 \mathrm{GHz}$. the resonant frequencies are adapted for $-25 \mathrm{~dB}$ at $2.7 \mathrm{GHz}$ and $-30 \mathrm{~dB}$ at $5 \mathrm{GHz}$ respectively.

Furthermore, we see the emergence of two frequency bands at 8 and $11 \mathrm{GHz}$ with very low adaptation. However, we believe that the appearance of these additional bands is attributed to the effect of the BNC connector. 


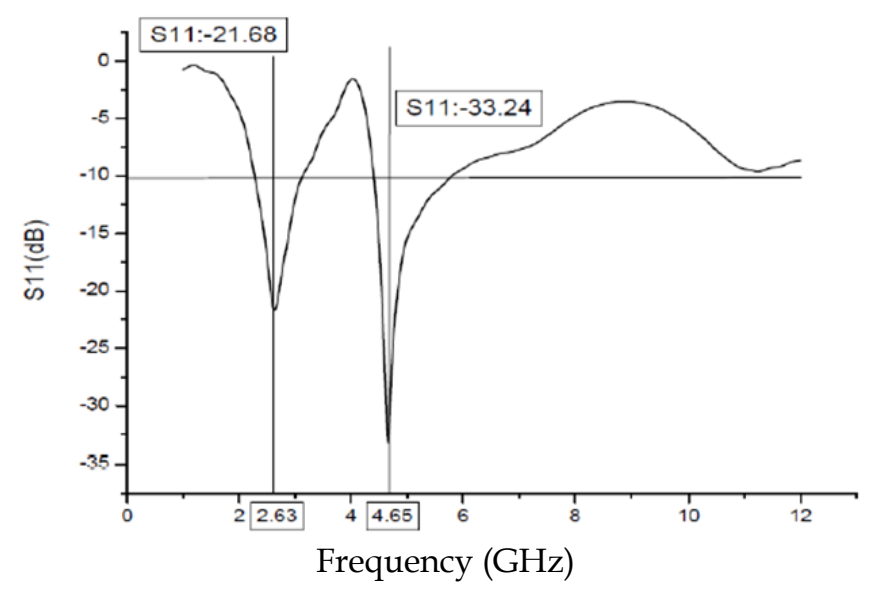

Figure 9. Evolution of the simulated reflection coefficient as a function of frequency.

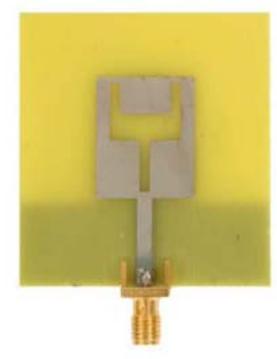

(a)

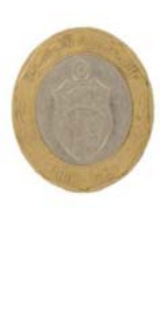

Figure 10. Photograph of the proposed dual band antenna: (a) Top view, (b) Side view.

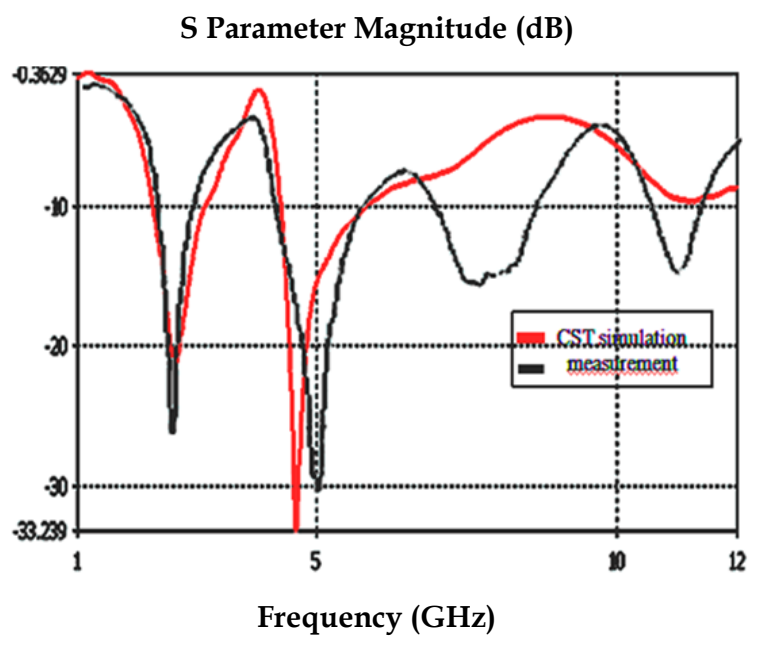

Figure 11. Simulated and measured reflection coefficient of the proposed antenna. 

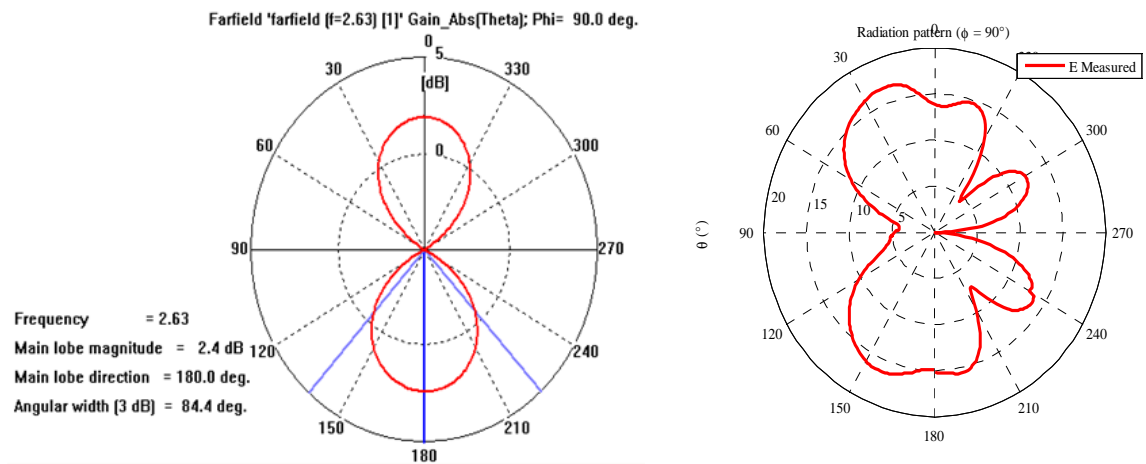

(a)
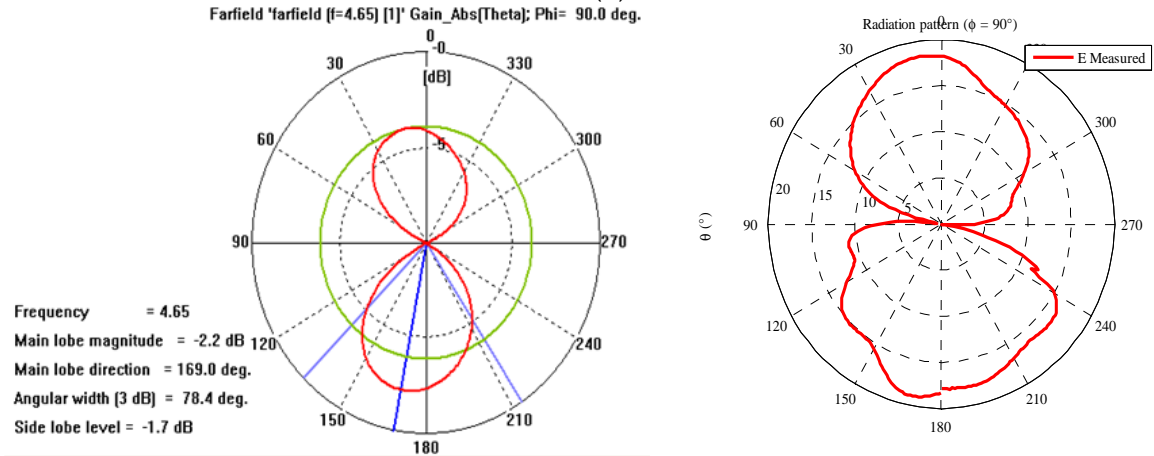

(b)

Figure 12. Simulated and measured radiation patterns of antenna in E-plane at (a) $2.63 \mathrm{GHz}$ and (b) $4.65 \mathrm{GHz}$.

\subsection{Radiation Patterns}

The simulated and measured E-plane (Copolarization) radiation patterns at resonant frequencies are shown in Figs. 12(a) and 12 (b), respectively.

The measurement results show an excellent agreement with the calculated data at the frequency $4.65 \mathrm{GHz}$. At $2.63 \mathrm{GHz}$, the measurement results are also correlated with calculations. The radiation patterns in E-plane are nearly bidirectional at $4.65 \mathrm{GHz}$, but slightly distorted at $2.63 \mathrm{GHz}$. However, the observed differences in the form of radiation patterns are substantially due to the differences in the amplitude scales in the plots.

For the simulated radiation pattern at 2.63 $\mathrm{GHz}$, the main lobe gain is $2.4 \mathrm{dBi}$ in perpendicular direction to the antenna surface (position $180^{\circ}$ in the plot) and the angular width is 84.4 degrees. But for the measured radiation pattern, the main lobe has an offset of $20^{\circ}$ from perpendicular direction (position $160^{\circ}$ in the plot) and the angular aperture is 70 degrees.
The radiation of the opposite side is also shifted by about 20 degrees.

From Fig. 12(b) we can see that the measured maximum side lobe level is $-1.2 \mathrm{dBi}$; the simulated maximum side lobe level is $-1.7 \mathrm{dBi}$. Note also that the main lobe magnitude for simulated radiation pattern is $-2.2 \mathrm{~dB}$ with an angular width of 78.4 degree in 169 degree of direction, which covered 90 degree in the same direction for the measured radiation patterns.

\section{Conclusions}

In this work, the new design of dual-band microstrip antenna has been successfully implemented and studied. This antenna is achieved by etching a Y-shaped slot matching concept on the radiating element and have been functioning in two bands $S$ and $C$. The electrical and radiation characteristics are given by the simulated and measured S-parameters and the radiation patterns of both bands. Good agreement between simulated and measured 
results has been achieved in the first operation band S. But, in the second operation band c, there is a slight difference explained by the insertion of the slot. This insertion creates other multi-resonant cavity, and each cavity has a new frequency band. The first band is appropriate for the original antenna and the other band is appropriate for the proposed antenna.

\section{References}

Abdelaziz AA (2006), Bandwidth enhancement of microstrip antenna. Progress In Electromagnetics Research, PIER 63:311-317.

Adnan S, Mustafa HB, Ucar, Yunus EE (2009), Loop-loaded printed dipole array design for a dual-band radar application. Antennas \& Propagation Conference, LAPC Loughborough. 529-532.

Adnen R, Ghnimi S, Ali G (2010, Electrical characteristics of a dual-band microstrip patch antenna for GSM/ UMTS / WLAN operations. International Journal of Communication Networks and Information Security (IJCNIS) 2(1):54-59.

Allen CM, Eldek AA, Elsherbeni AZ, Smith CE (2005), Dual tapered meander slot antenna for radar applications. IEEE transactions on antennas and propagation 53(7):23242328.

BALANIS CA (1997), Antenna theory: analysis and design. $2^{\text {nd }}$ Edition, John Wiley \& Sons, New York, USA.

Christina F, Jou JWW, Chien-Jen W (2009), Novel broadband monopole antennas with dual-band circular polarization. IEEE transactions on antennas and propagation 57(4):1027-1034.
Gai S, Jiao YC, Yang YB, Li CY, Gong JG (2010), Design of a novel microstrip-fed dual-band slot antenna for WLAN applications. Progress In Electromagnetics Research Letters 13:7581.

Ghnimi S, Rawia W, Ali G, Razban T (2013), A new design of an $S / X$ dual band circular slot antenna for radar applications. Journal of Microwave Power and Electromagnetic Energy 47(2):138-146.

Khaled EEM, Saad AAR (2008), Multiwide band compact microstrip patch antenna based on slot matching. Progress In Electromagnetics Research C 4:169-177.

Khunead G, Nakasuwan J, Songthanapitak $\mathrm{N}$, Anantrasirichai N (2007), Investigate rectangular slot antenna with L-shaped strip. Piers online 3(7):1076-1079.

Qu X, Zhong S, Zhang YM (2006), Dualband dual-polarised microstrip antenna array for SAR applications. Electronic letters 42(24):1376-1377.

Saidatul NA, Azremi AAH, Ahmad RB, Soh PJ, Malek F (2009), Multiband fractal planar inverted $\mathrm{F}$ antenna (F-PIFA) for mobile phone application. Progress In Electromagnetics Research B 14:127-148.

Zhilong $\mathrm{M}$, Cheng $\mathrm{H}$, Qin $\mathrm{Z}$, Mingbo $\mathrm{P}$, Xiaoliang M, Xiangang L (2012), Design of a patch antenna with dual-band radar cross section reduction. International Conference on Microwave and Millimeter Wave Technology (ICMMT) 5:1-3. 